\title{
Neuropathic pain associated with mucocele in the maxillary sinus
}

\author{
A López-Valverde ${ }^{1}$, R Gómez de Diego ${ }^{1}$, J Montero ${ }^{2}$, A Albaladejo ${ }^{2}$ \\ ${ }^{1}$ Associate Professor. Department of Surgery. Faculty of Medicine. University of Salamanca. Spain. \\ ${ }^{2}$ Tenured lecturer. Department of Surgery. Faculty of Medicine. University of Salamanca. Spain.
}

Correspondence:

Antonio López Valverde.

Facultad de Medicina y Odontología, Departamento de Cirugía.

C/Alfonso X el Sabio S/N. C.P. 37007.

Salamanca. Spain

E-mail:alopezvalverde@gmail.com

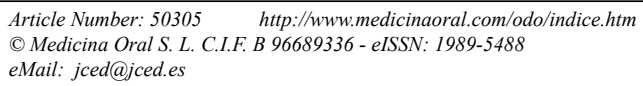

\begin{abstract}
Mucocele in the paranasal sinuses consists of a clinically unpainful, pseudocystic lesion that may be confused with cysts with mucosal accumulation due to an obstruction of a seromucinous gland or with other lesions displaying expansive masses in the maxillary sinuses. Histologically, mucocele consists of an accumulation of seromucosal secretion lined by epithelium. It is an infrequent pathology, whose treatment has evolved favourably to a considerable extent over the past decades. Here we report a case of mucocele located in a paranasal sinus associated with a syndrome of odontalgic neuritic pain. After the collection of complementary radiological evidence, antrostomic surgical treatment was implemented on the lateral-external wall of the maxillary sinus with a view to fully removing the cystic capsule, after which a histopathological study of the sample was performed. The lesion and the neuropathic pain disappeared following removal of the cystic mass, and after 21 months of follow-up the patient has remained free of relapse. Conclusions: lateral antrostomy for the removal of mucocele in the maxillary sinus can alleviate the neuropathic pain possibly associated with the pathology.
\end{abstract}

Key words: Paranasal mucocele, sinus antrostomy, neuritic pain. 


\section{Introduction}

Mucocele is a chronic expansive pseudocystic lesion of the mucosa that may appear in the paranasal sinuses. It is benign and curable and tends to be clinically silent for a long time, affecting any individual regardless of his or her age or sex. It behaves as a mass that tends to swell, and is filled with mucus or mucoid exudates limited by the mucoperiostium that surrounds the cranial sinuses. Because they are expansive, maxillary paranasal pseudotumours due to the accumulation of mucous secretion are able to produce osteolysis in the underlying bone due to compression, the percentage of sinus volume affected and the increase in pressure generated by the mucus being associated with this (1). A mucocele may become infected and give rise to a mucopiocele, with greater destructive capacity (2).

The most frequent causal factor is infectious rhinosinusal disease, either alone or concomitant with inflammatory and allergic factors (3); early diagnosis and treatment are of crucial importance.

The radiographic aspect of a mucocele is that of a homogeneous hypodense mass, with no air and usually with semi-lunar contours, that expands through the maxillary sinus. Computerized tomography (CT) is a suitable complementary radiographic test for analysis of the extent of the lesion (4). In order to avoid relapses, surgery -via endoscopy or antrostomy of the lateral wall of the maxillary sinus- must remove the entire cystic capsule of the mucocele (5).

\section{Case report}

The patient was a man of 37 who had a history of persistent nasal obstruction, rhinorrhea, pain in his hemimaxillary molar teeth, and pain through the upper right quadrant of his head. He was diagnosed, and treated unsuccessfully, as having chronic sinusitis, for which he received different treatments with antibiotics and nasal decongestants (6). In view of the persistence of his neu- ralgic symptoms, he was referred to his dentist in order to obtain a diagnosis to exclude any kind of dental pathology. In recurring spontaneous odontalgias, regional hyperalgesia may occur due to central sensitization (autonomous nervous system), with regional vasomotor disorder (7).

After his clinical background had been recorded (with no findings of interest) and both extraoral and intraoral examinations were performed, the presence of dental alterations, anomalies, or pathology was ruled out, and complementary radiological tests were requested. CT revealed the presence of an expansive cystic mass in the maxillary sinus, extending towards the medial wall and containing an irregular mass of greater radio-opaqueness with radiolucent inclusions and some calcifications (Fig. 1A, 1B), together with a slight vestibular swelling at the base of the zygomatic bone (Fig. 2A, 2B).

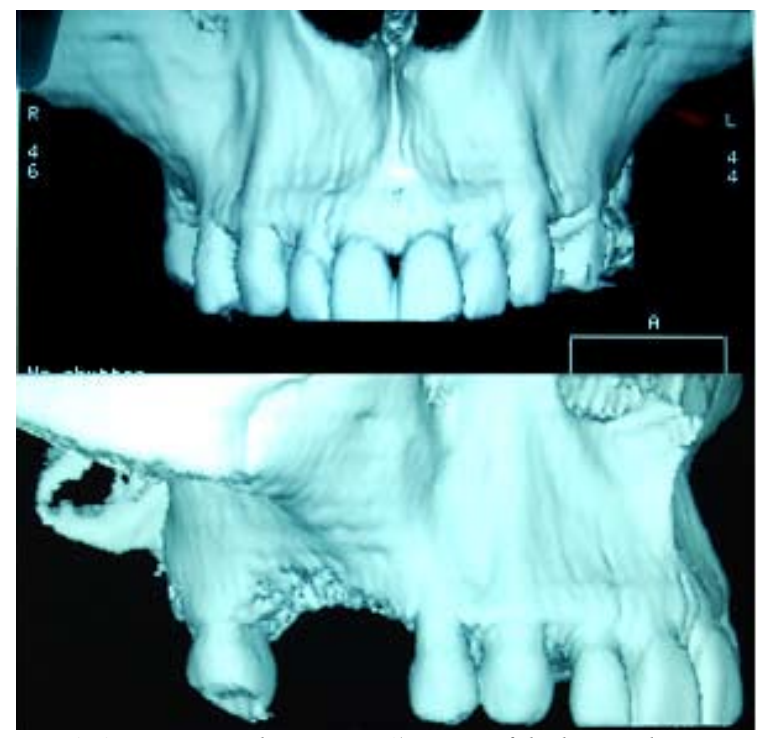

Fig. 2. 3-D tomographic image: A) image of the bone volume, showing bones of high density with an erosive picture associated with the edentulate alveolar ridge; B) right zygomatic bone showing bone remodelling associated with the cystic mass.
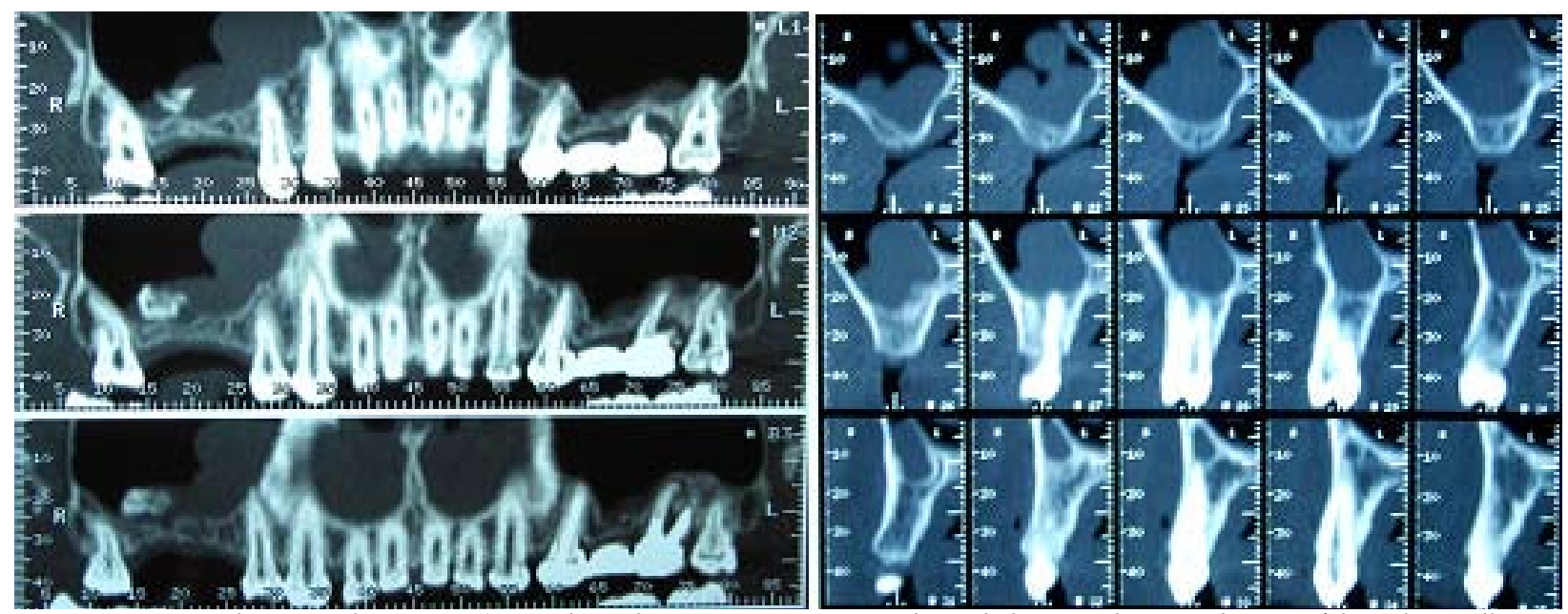

Fig. 1. Computerized tomographic image: A) irregular radio-opaque occupation with a radiolucent inclusion in the area of the right maxillary sinus; B) Tomographic sections of the occupied sinus, showing an irregular mass occupying the caudal portion and affecting the wall of the nose. 


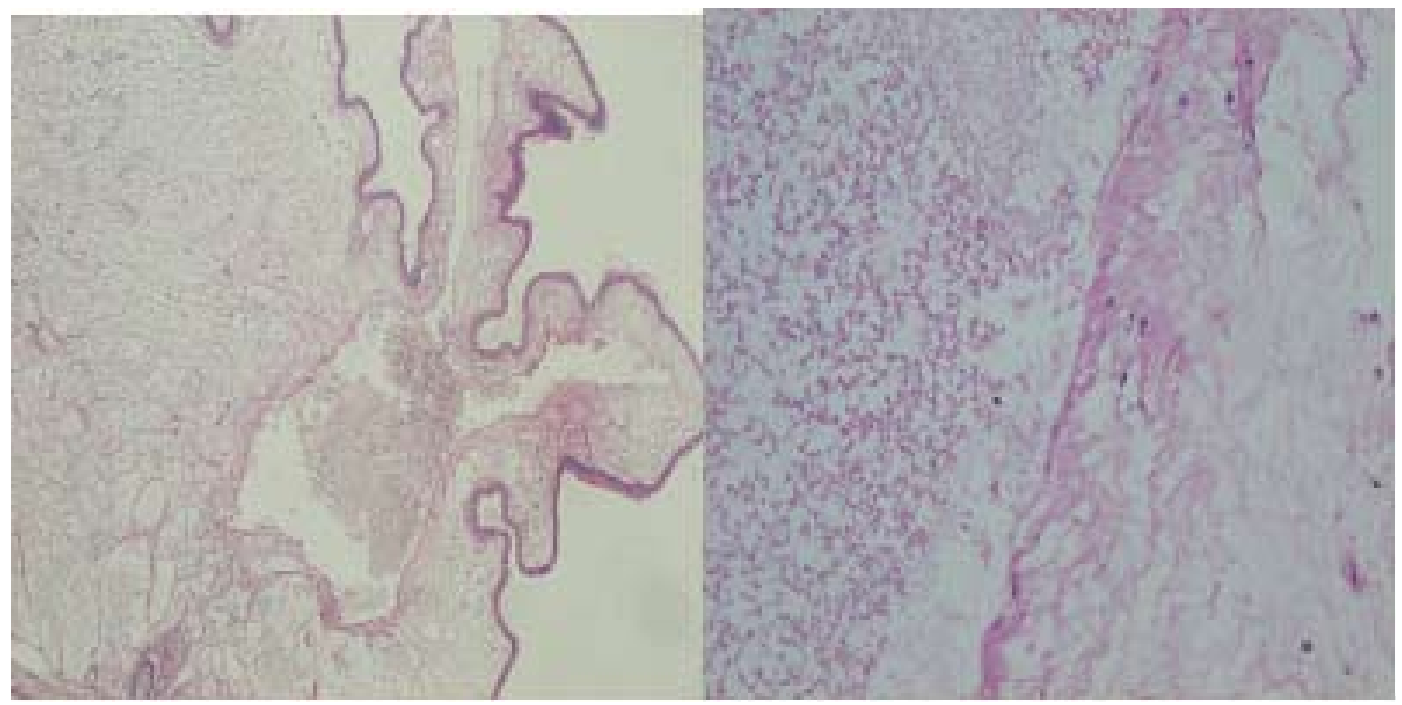

Fig. 3. Histological image of the mucocele: A) wall formed by hyaline connective tissue, with cystic areas lined by columnar epithelium. Hematoxiline-eosine (H-E) 10x; B) detail of wall, showing the characteristics of the connective tissue with hyaline areas and other more lax areas. H-E.20x.

The patient agreed to undergo surgical treatment via antrostomy of the latero-external wall of the maxillary sinus. The surgical intervention consisted of complete removal of the cystic mass with total obliteration of the Schneider membrane and reconstruction of the antrostomized wall with a reabsorbable membrane of long-term type I and III collagen (BioGuideTM) (Gesitlich, Wolhusen, Switzerland).

Following fixation of the material removed in $10 \%$ formaldehyde and later staining with haematoxylin-eosin, an anatomopathological analytical study confirmed the diagnosis of maxillary mucocele.

The post-operative evolution of the patient, with no rhinorrhea or nasal bleeding, was satisfactory and his neuralgia disappeared immediately after the intervention. Currently, after 21 months, the patient remains symptom-free and his earlier problems of vasomotor effects, trophic changes and headaches have disappeared completely.

\section{Discussion}

The formation of paranasal mucoceles has been widely described in the literature, although their physiopathology is still being debated $(8,9)$. Owing to their disposition, fronto-ethmoidal mucoceles are the commonest type $(80 \%)$, followed by those developing in the maxillary sinuses $(17 \%)$, whereas only $3 \%$ are found in the sphenoidal sinuses (10). They are benign lesions, with an identical frequency of occurrence in adults of both sexes, their prevalence being higher between the third and fourth decades of life (11). They are extremely rare in children and adolescents (12).

It is necessary to establish a differential diagnosis with cysts involving the accumulation of mucus due to the obstruction of a seromucinous gland, antrochoanal po- lyps, aspergillar sinusitis, and above all epidermoid, adenocystic and mucoepidermoid carcinomas.

Treatment of these lesions is simple removal, using the most atraumatic approach possible but one that will ensure complete enucleation of the pseudocyst. Most authors have confirmed that treatment should ideally be performed by endoscopy owing to its reduced invasiveness and short recovery time $(5,13)$.

Histological mucoceles exhibit a respiratory epithelium lining the pseudocyst that sometimes evolves to a squamous metaplasia, which would account for the alterations in mucociliar flow and the accumulation of mucus (8).

Pain elicited by the presence of a mucocele tends to be rare, but when it is present it is generally of the somatic type, deep, visceral, and its nature is clearly interoceptive, characteristic of the mucosa of the paranasal sinuses, the pharynx and nasal cavity (9). Although Schneider's membrane is resistant to painful stimuli and is almost insensitive to pain owing to the absence of nociceptive terminals, where it occurs the pain stems from the zone of drainage (ostium) from the maxillary sinus to the nasal fossae and induces a nociception affecting the upper molars and face (14). A differential diagnosis should be performed with peripheral neuritis (alteration of the afferent nerve fibres) as a result of inflammation of the pterygo-maxillary plexus as a consequence of tumefaction of the sinus membrane, with the presence of pain in the upper homolateral teeth; this pain is heterotopic and projects to the peripheral distribution of the affected nerve, in the territory of the maxillary branch (V2) of the trigeminal nerve (15). This neuropathic pain is usually accompanied by symptoms such as hyperesthesia or paraesthesia of the maxillary teeth as a consequence of the affectation of the sinus. As summary we could conclude 
that lateral antrostomy for the removal of mucoceles in the paranasal sinuses can alleviate the neuropathic pain possibly associated with this pathology.

\section{References}

1. Peral Cagigal B, Barrientos Lezcano J, Floriano Blanco R, García Cantera JM, Sánchez Cuéllar LA, Verrier Hernández A. Frontal sinus mucocele with intracranial and intraorbital extension. Med Oral Patol Oral Cir Bucal. 2006;11:527-30.

2. Atherino CC, Atherino TC. Maxillary sinus mucopyoceles. Arch Otolaryngol. 1984. 110:200-2.

3. DelGaudio JM, Evans SH, Sobol SE, Parikh SL. Intracranial complications of sinusitis: what is the role of endoscopic sinus surgery in the acute setting. Am J Otolaryngol. $2010 ; 31: 25-8$.

4. Weber R, Draf W, Kratzsch B, Hosemann W, Schaefer SD. Modern concepts of frontal sinus surgery. Laryngoscope. 2001; 111:137-46.

5. Bockmühl U, Kratzsch B, Benda K, Draf W. Surgery for paranasal sinus mucocoeles: efficacy of endonasal micro-endoscopic management and long-term results of 185 patients Rhinology. 2006; 44: 62-7. 6 . Kinney WC. Rhinosinusitis treatment protocol: changing provider habits in primary care. Otolaryngol Head Neck Surg. 2002;126: 61422 .

7. Gross D. Pain and autonomic nervous system. Hippokrates. 1969; 40:168-75.

8. Lund VJ. Anatomical considerations in the aetiology of frontoethmoidal mucoceles. Rhinology. 1987;25:83-8.

9. Busaba NY, Salman SD. Maxillary sinus mucoceles: clinical presentation and long-term results of endoscopic surgical treatment. Laryngoscope. 1999;109:1446-9.

10. Gardner DG, Gullane PJ. Mucoceles of the maxillary sinus. Oral Surg Oral Med Oral Pathol. 1986;62:538-43.

11. Ramesh A, Pabla T. Mucous retention cyst of maxillary sinuses. J Mass Dent Soc. 2008;57:14-5.

12. Olze H, Matthias C, Degenhardt P.Paediatric paranasal sinus mucoceles. Eur J Pediatr Surg. 2006;16:192-6.

13. Mohammadi G, Sayyah Meli MR, Naderpour M. Endoscopic surgical treatment of paranasal sinus mucocele. Med J Malaysia. 2008;63:39-40.

14. Chow JM. Rhinologic headaches. Otolaryngol Head Neck Surg. 1994; 111: 211-8.

15. Ence BK, Parsons DS. Mucous retention cyst causing pain of the infraorbital nerve. Otolaryngol Head Neck Surg. 1990;103:1031-4. 